\title{
Incremental approximation computation in incomplete ordered decision systems
}

\author{
Guanglei Gou ${ }^{1,2}$, Guoyin Wang ${ }^{3, *}$ \\ ${ }^{1}$ School of Information Science and Technology, Southwest Jiaotong University, \\ Chengdu 610031, P.R. China \\ ${ }^{2}$ School of Computer Science and Engineering Chongqing University of Technology, \\ Chongqing 400065, P.R. China \\ E-mail: ggl@cqut.edu.cn \\ ${ }^{3}$ Chongqing Key Laboratory of Computational Intelligence, Chongqing University of Posts and \\ Telecommunications, \\ Chongqing 400065, P.R. China \\ E-mail: wanggy@cqupt.edu.cn
}

Received 24 October 2016

Accepted 17 December 2016

\begin{abstract}
Approximation computation is a critical step in rough sets theory used in knowledge discovery and other related tasks. In practical applications, an information system often evolves over time by the variation of attributes or objects. Effectively computing approximations is vital in data mining. Dominance-based rough set approach can handle information with preference-ordered attribute domain, but it is not able to handle the situation of data missing. Confidential Dominance-based Rough Set Approach (CDRSA) is introduced to process Incomplete Ordered Decision System (IODS). This paper focuses on incremental updating approximations under dynamic environment in IODS. With the CDRSA, the principles of incremental updating approximations are discussed while the variation of attribute sets or the union of subsets of objects and the corresponding incremental algorithms are developed. Comparative experiments on data sets of UCI and results show that the proposed incremental approaches can improve the performance of updating approximations effectively by a significant shortening of the computational time.
\end{abstract}

Keywords: Incomplete Ordered Decision Systems, Confidential dominance relation, Approximations, Incremental updating

\section{Introduction}

Rough set theory is proposed by Pawlak ${ }^{1}$ to deal with inconsistency problems, which is useful in fields such as knowledge discovery $2,3,4$, decision analysis $^{5,6}$, data mining ${ }^{7,8}$, etc. Classic Rough Set (CRS) is based on equivalence relation, which is used to deal with discrete attributes values. However, CRS can not be able to discover uncertainty from attributes with preference order domains, which is important to the multi-criteria decision analysis, e.g., risk evaluation, pollution rating. In order to solve this problem, Greco, Matarazzo and Słowinski proposed Dominance-

\footnotetext{
* Corresponding author.
} 
based Rough Set Approach (DRSA), which is based on dominance relation instead of equivalence relation $^{9,10}$. DRSA attracted much attention in recent years, and has been applied to multi-criteria classification $^{11}$, attribute reduction ${ }^{12,13}$, customer behavior prediction ${ }^{14}$, bankrupt risk prediction ${ }^{15}$, water quality evaluation ${ }^{16}$, etc.

DRSA has been introduced to handle the crisp ordered information system, however, Real life applications face data missing due to various causes. Greco et al. extended dominance relation, which requires the referent object has no missing data, to deal with missing data in rough set analysis of multiattribute and multi-criteria decision problem ${ }^{17,18}$. Błaszczynski et al. discussed different ways of handling missing values in sorting problems with monotonicity constraints in DRSA ${ }^{19}$. He et al. investigated an extend dominance relation to discover knowledge from approximations ${ }^{20}$. Shao and Zhang applied extended DRSA to reasoning, rule extracting and knowledge reduction in incomplete ordered information system ${ }^{21}$. In order to avoid the comparison of two objects have no common known attribute value, $\mathrm{Hu}$ and Liu discussed a limited extended dominance $^{22}$ and generalized extended dominance relation ${ }^{23}$. Generalized extended dominance relation contains limited extended dominance by chosen the parameter. Chen et al. studied $k$-degree extended dominance characteristic ( $k$-degree EDCR), which is considering tow case of missing data that are "do not known" and " do not care" 24 . Du et al. proposed the characteristic-based dominance relation to deal with incomplete ordered information ${ }^{25}$. Yang et al. proposed a similarity dominance relation and studied knowledge reduction under incomplete ordered information system ${ }^{26}$. Luo et al. combined the limited extended dominance relation with similarity dominance relation and proposed a limited extended dominance relation considering maximum and minimum values in dominating or dominated relation when comparing their lost ${ }^{27}$. It is too strict to be practical because maximum and minimum attribute values are hard to known. Yang et al. introduced a valued dominance relation considering the probability that an object is dominating another one by using the information given unknown values ${ }^{28}$. In order to avoid semantic contradiction on the ordered relation, Gou et al. proposed confidential dominance relation to extend DRSA to deal with missing data in Incomplete Ordered Decision System (IODS) ${ }^{29}$.

The information system commonly evolves with time, typically, the set of attributes, the set of objects and attribute values may dynamically change. The approximations may alter by the variation of the information system. The traditional methodologies to update approximations are inefficient because they need to recomputing from scratch. Incremental update is a feasible and effective in processing dynamic information since previous data structures and knowledge are optimized for updating approximations. In the case of variation of attributes, Chan proposed an incremental learning method for maintaining approximations in CRS by added into or deleted from one attribute ${ }^{30}$. Li et al. proposed an incremental approach for updating approximations under rough set based the characteristic relation when adding or removing some attributes in incomplete information system ${ }^{31}$. Zhang et al. presented matrix approaches for dynamic attribute variation in set-valued information system ${ }^{32}$. Li et al. studies the principle of updating dominating sets and dominated sets and proposed incremental approaches for updating approximations ${ }^{33}$. Zhang et al. investigated incremental updating of rough approximations in interval-valued information systems ${ }^{34}$. Liu et al. presented approaches for incremental updating approximations in probabilistic rough sets under the variation of attributes ${ }^{35}$. Yang et al. designed fast algorithms for updating the multigranulation rough approximations with increasing of the granular structures ${ }^{36}$. In the case of variation of objects, Shan et al. introduced an incremental modification for classification rules ${ }^{37}$. Zheng and Wang developed a rough set and rule tree based incremental knowledge acquisition algorithm, which updates rules by adding and pruning the rule tree incrementally ${ }^{38}$. Zhang et al. designed parallel algorithms based on MapReduce for calculating approximations of rough sets ${ }^{39,40}$. Luo et al. proposed a matrix approach to decision-theoretic rough sets for evolving data ${ }^{41}$. Luo et al. presented efficient updating of probabilistic approximations with incre- 
mental objects ${ }^{42}$. Li et al. developed incremental algorithms for updating approximations of composite rough sets when adding or removing some objects ${ }^{43}$. $\mathrm{Li}$ et al. studied the dynamic maintenance of approximations in DRSA when one object is added or removed $^{44}$, furthermore, parallel algorithms are developed to speed up computing approximations in DRSA $^{45}$. Błaszczynski and Słowinski proposed an incremental algorithm for induction rules based on the Apriori under the variable consistency DRSA ${ }^{46}$. Wang et al. presented efficient updating rough approximations with multi-dimensional variation of ordered data ${ }^{47}$. In the case of variation of attributes values, Zeng et al. developed dynamical updating fuzzy rough approximations for hybrid data under the variation of attribute values ${ }^{48}$. Chen et al. proposed an incremental method for updating approximations while objects dynamically alter and attributes values vary in variable precision rough set model $^{49}$. And Chen et al. discussed the principles of maintenance of approximations and algorithms for incremental updating approximations are designed when attribute's values coarsening or refining in IODS ${ }^{24}$. However, incremental approaches for updating approximations under IODS when the variation of attributes or objects have not been taken into account until now. In real-life application, such as monitoring domain, attributes and objects are always dynamic changed instead of attribute values. Thus, this paper focuses on incremental methods for updating approximations of confidential dominancebased rough set under the variation of attributes or objects in IODS.

The paper is organized as follows. In Section 2, basic concepts of IODS and dominance relation are reviewed and confidential dominance-based rough set approach is introduced. In Section 3, we discuss the principles of incremental updating approximations and propose algorithms when some attributes are added into or deleted from. In Section 4, incremental approach for updating approximations is given when subsets of objects are merged. In Section 5, some experiments are conducted to evaluate the performance of proposed approaches. The paper ends with conclusion and future work in Section 6.

\section{Confidential Dominance-based Rough Set Approach}

In this section, some basic concepts of decision systems, including IODS, confidential dominate relation are introduced.

Definition $1{ }^{24} A$ decision system is a 4-tuple $I O D S=(U, A, V, f) . U$ is a finite non-empty set of objects, called the universe. $A$ is a non-empty finite set of attributes, $A=C \cup d$ where $C$ and $d$ denote the sets of condition attributes and decision attribute. $V$ is a domain of attributes. The domain of $C$ and $d$ is ranked according to an increasing or decreasing preference. The attributes are criteria. $f: U \times A \rightarrow V$ is an information function. $f=\left\{f\left(x_{i}, a\right) \mid f\left(x_{i}, a\right): x_{i} \rightarrow v_{a}, a \in C, x_{i} \in U, 1 \leqslant\right.$ $i \leqslant|U|\}$, where $|\cdot|$ denotes the cardinality of a set. $f\left(x_{i}, a\right)=v_{a}(1 \leqslant i \leqslant|U|)$ denotes the attribute value of object $x_{i}$ under $a$. If all attributes? value are know in the decision system, it is a complete ordered decision system. If exists any missing data, it is an incomplete ordered decision system (IODS). All the missing values are denoted by "*”.

Definition $2{ }^{9,10}$ Let $x, y \in U, P \subseteq C$. If $\forall a \in P$, $f(y, a) \succeq f(x, a)$ then we denote $y D_{P} x$. The relation is called a dominance relation. Here, $f(y, a) \succeq$ $f(x, a)$ means $y$ at least as good as (outranks) $x$ with respect to criterion $a$, which is defined by $f(y, a) \geqslant$ $f(x, a)$ according to increasing preference ordering, and $f(y, a) \leqslant f(x, a)$ for decreasing ordering.

Definition 3 Let IODS $=(U, A, V, f), \quad P \subseteq C$, $B_{P}(x)=\{b \mid b \in P \wedge f(x, b) \neq *\}$. A confidential dominance relation $(C D R)$ is defined as follows.

$$
\begin{aligned}
C D R(P)= & \left\{(x, y) \in U^{2} \mid\left(B_{P}(x) \subseteq B_{P}(y)\right)\right. \\
& \wedge \forall_{q \in P}((f(x, q)=* \wedge f(y, q)=*) \vee \\
& (f(x, q)=* \wedge f(y, q) \neq *) \vee f(y, a) \succeq f(x, a)\}
\end{aligned}
$$

$y D_{P}^{C D R} x$ denotes that $y$ is dominates $x$ in CDR, which means the object $y$ is more detailed than referent $x$, and $y$ is weakly preferred over $x$ with respect to each criterion $q$ for which the evaluation of referent $\mathrm{x}$ is known.

Definition 4 For $P \subseteq C, x \in U, D_{P}^{C D R+}(x)=\{y \in$ $\left.U \mid y D_{P}^{C D R} x\right\}$ is $P$ confidential dominating set of $x$. 
$D_{P}^{C D R-}(x)=\left\{y \in U \mid x D_{P}^{C D R} y\right\}$ is $P$ confidential dominated set of $x$.

The set of decision attributes $d$ partitions $U$ into a finite number of classes. Let $\mathrm{Cl}=\left\{C l_{t} \mid t \in\right.$ $\{1,2, \ldots, n\}\}$, where $C l_{n} \succ C l_{n-1} \succ \ldots \succ C l_{1}$. An upward union and downward union of classes are defined respectively as follows: $C l_{t}^{\geqslant}=\bigcup_{s \geqslant t} C l_{s}, C l_{t}^{\leqslant}=$ $\bigcup_{s \leqslant t} C l_{s}$, where $t, s \in\{1,2, \ldots, n\} . x \in C l_{t}^{\geqslant}$means $x$ at least belongs to class $C l_{t}$, while $x \in C l_{t}^{\leqslant}$means $x$ at most belongs to class $C l_{t}$

Definition 5 Let IODS $=(U, A, V, f), P \subseteq C, x \in U$. The $P$ upper and lower approximations of $C l_{t}^{\geqslant}$and $\mathrm{Cl}_{t}^{\leqslant}$under the CDR are defined respectively as follows.

$$
\begin{gathered}
\underline{P}\left(C l_{t}^{\geqslant}\right)=\left\{x \in U \mid D_{P}^{C D R+}(x) \subseteq C l_{t}^{\geqslant}\right\} \\
\bar{P}\left(C l_{t}^{\geqslant}\right)=\bigcup_{x \in C l_{t}^{\geqslant}} D_{P}^{C D R+}(x)=\left\{x \mid D_{P}^{C D R-}(x) \cap C l_{t}^{\geqslant} \neq \emptyset\right\} \\
\underline{P}\left(C l_{t}^{\leqslant}\right)=\left\{x \in U \mid D_{P}^{C D R-}(x) \subseteq C l_{t}^{\leqslant}\right\} \\
\bar{P}\left(C l_{t}^{\leqslant}\right)=\bigcup_{x \in C l_{t}^{\leqslant}} D_{P}^{C D R-}(x)=\left\{x \mid D_{P}^{C D R+}(x) \cap C l_{t}^{\leqslant} \neq \emptyset\right\}
\end{gathered}
$$

The boundary region is defined as follows.

$$
\begin{aligned}
& B n_{P}\left(C l_{t}^{\geqslant}\right)=\bar{P}\left(C l_{t}^{\geqslant}\right)-\underline{P}\left(C l_{t}^{\geqslant}\right) \\
& B n_{P}\left(C l_{t}^{\lessgtr}\right)=\bar{P}\left(C l_{t}^{\leqslant}\right)-\underline{P}\left(C l_{t}^{\lessgtr}\right)
\end{aligned}
$$

The objects in the boundary region are inconsistent, which means an object $x$ is confidential dominating another object $y$, however, $x$ is assigned to a class worse than $y$.

Definition 6 For any $x \in U$ in an IODS = $(U, A, V, f), \quad \delta(x)=<l_{P}(x), u_{P}(x)>$ is called $P$ generalized decision of the object $x$, where $l_{P}(x)=\min \left\{t \mid D_{P}^{C D R+}(x) \cap C l_{t} \neq \emptyset\right\}$ and $u_{P}(x)=$ $\max \left\{t \mid D_{P}^{C D R-}(x) \cap C l_{t} \neq \emptyset\right\}$.

Property 1 Let IODS $=(U, A, V, f), P \subseteq C$. For any $x \in U$, the following items hold.

(1) if $l_{P}(x) \geqslant C l_{t}$, then $x \in \underline{P}\left(C l_{t}^{\geqslant}\right)$

(2)if $u_{P}(x) \leqslant C l_{t}$, then $x \in \underline{P}\left(C l_{t}^{\leqslant}\right)$

(3)if $l_{P}(x) \leqslant C l_{t}$, then $x \in \bar{P}\left(C l_{t}^{\leqslant}\right)$

(4)if $u_{P}(x) \geqslant C l_{t}$, then $x \in \bar{P}\left(C l_{t}^{\geqslant}\right)$
Proof. (1) $U$ is partitioned by $d$ into decision classes $C l=\left\{C l_{t} \mid t \in\{1,2, \ldots, n\}\right\}$. Since $l_{P}(x)=$ $\min \left\{t \in\{1,2 \ldots, n\}: D_{P}^{C D R+}(x) \cap C l_{t}\right\}$, for $\forall y \in$ $D_{P}^{C D R+}(x)$ we have $f(y, d) \geqslant C l_{t}$ if $l_{P}(x) \geqslant t$. Hence, $D_{P}^{C D R+}(x) \subseteq C l_{t}^{\geqslant} \Rightarrow x \in \underline{P}\left(C l_{t}^{\geqslant}\right)$.

(2)-(4) are similar to (1).

According to Property 1, approximations of $\mathrm{Cl}_{t}^{\geqslant}$ and $C l_{t}^{\leqslant}$under the CDR are defined as follows.

$$
\begin{aligned}
& \underline{P}\left(C l_{t}^{\geqslant}\right)=\left\{x \in U \mid l_{P}(x) \geqslant C l_{t}\right\} \\
& \underline{P}\left(C l_{t}^{\lessgtr}\right)=\left\{x \in U \mid u_{P}(x) \leqslant C l_{t}\right\} \\
& \bar{P}\left(C l_{t}^{\geqslant}\right)=\left\{x \in U \mid u_{P}(x) \geqslant C l_{t}\right\} \\
& \bar{P}\left(C l_{t}^{\lessgtr}\right)=\left\{x \in U \mid l_{P}(x) \leqslant C l_{t}\right\}
\end{aligned}
$$

Example 1 Let us consider an incomplete ordered decision system $I O D S=(U, A, V, f)$ presented in Table 1, where $U=\left\{x_{1}, x_{2}, \cdots, x_{10}\right\}, A=C \cup d$, $C=\left\{a_{1}, a_{2}, a_{3}\right\}, V_{d}=\{1,2,3,4\}$.

Table 1. An IODS

\begin{tabular}{ccccc}
\hline & $a_{1}$ & $a_{2}$ & $a_{3}$ & $d$ \\
\hline$x_{1}$ & 4 & 4 & 3 & 4 \\
$x_{2}$ & 3 & 2 & 3 & 3 \\
$x_{3}$ & 4 & $*$ & 2 & 3 \\
$x_{4}$ & 2 & 2 & 2 & 2 \\
$x_{5}$ & 2 & 1 & 2 & 2 \\
$x_{6}$ & 3 & 1 & 2 & 1 \\
$x_{7}$ & $*$ & 2 & 2 & 2 \\
$x_{8}$ & 4 & 1 & 2 & 2 \\
$x_{9}$ & 3 & $*$ & 2 & 3 \\
$x_{10}$ & 4 & 3 & 3 & 3 \\
\hline
\end{tabular}

From Table 1, upward union of decision is calculated as follows.

$$
\mathrm{Cl}_{4}^{\geqslant}=\left\{x_{1}\right\}, \mathrm{Cl}_{3}^{\geqslant}=\left\{x_{1}, x_{2}, x_{3}, x_{9}, x_{10}\right\},
$$
$C l_{2}^{\geqslant}=\left\{x_{1}, x_{2}, x_{3}, x_{4}, x_{5}, x_{7}, x_{8}, x_{9}, x_{10}\right\}, C l_{1}^{\geqslant}=U$.

$C$ confidential dominating sets are calculated as follows.

$$
\begin{aligned}
& \quad D_{C}^{C D R+}\left(x_{1}\right)=\left\{x_{1}\right\}, D_{C}^{C D R+}\left(x_{2}\right)=\left\{x_{1}, x_{2}, x_{10}\right\}, \\
& D_{C}^{C D R+}\left(x_{3}\right)=\left\{x_{1}, x_{3}, x_{8}, x_{10}\right\}, \quad D_{C}^{C D R+}\left(x_{4}\right)= \\
& \left\{x_{1}, x_{2}, x_{4}, x_{10}\right\}, \\
& D_{C}^{C D R+}\left(x_{5}\right)=\left\{x_{1}, x_{2}, x_{4}, x_{5}, x_{6}, x_{8}, x_{10}\right\}, D_{C}^{C D R+}\left(x_{6}\right)=
\end{aligned}
$$


$\left\{x_{1}, x_{2}, x_{6}, x_{8}, x_{10}\right\}, D_{C}^{C D R+}\left(x_{7}\right)=\left\{x_{1}, x_{2}, x_{4}, x_{7}, x_{10}\right\}$, $D_{C}^{C D R+}\left(x_{8}\right)=\left\{x_{1}, x_{8}, x_{10}\right\}$,

$D_{C}^{C D R+}\left(x_{9}\right)=\left\{x_{1}, x_{2}, x_{3}, x_{6}, x_{8}, x_{9}, x_{10}\right\}, D_{C}^{C D R+}\left(x_{10}\right)=$ $\left\{x_{1}, x_{10}\right\}$.

Thus, approximations of upward union $C l_{t}^{\geqslant}$with respect to $C$ confidential dominating sets are computed as follows.

$$
\underline{C}\left(C l_{4}^{\geqslant}\right)=\left\{x_{1}\right\}, \underline{C}\left(C l_{3}^{\geqslant}\right)=\left\{x_{1}, x_{2}, x_{10}\right\},
$$

$\underline{C}\left(C l_{2}^{\geqslant}\right)=\left\{x_{1}, x_{2}, x_{3}, x_{4}, x_{7}, x_{8}, x_{10}\right\}, \underline{C}\left(C l_{1}^{\geqslant}\right)=U$.

$\bar{C}\left(C l_{4}^{\geqslant}\right)=\left\{x_{1}\right\}, \bar{C}\left(C l_{3}^{\geqslant}\right)=\left\{x_{1}, x_{2}, x_{3}, x_{6}, x_{8}, x_{9}, x_{10}\right\}$, $\bar{C}\left(C l_{2}^{\geqslant}\right)=U, \bar{C}\left(C l_{1}^{\geqslant}\right)=U$.

Analogously, approximations of $C$ confidential dominated set with respect to upward union $\mathrm{Cl}_{t}^{\leqslant}$can be calculated. For simplicity and clarity, the following examples discuss CDRSA only with the increasing preference order.

\section{Incremental updating approximations under the variation of attribute sets}

Approximations computing is a critical step for applying CDRSA in knowledge discovery. Traditional approach of approximations computing is time-consuming when attributes are changed because it should recompute from scratch. In this section, approaches of incremental updating approximations under the variation of attributes are discussed.

\subsection{Incremental updating approximations when new attributes added}

Lemma 1 Let IODS $=(U, A, V, f), P \subset C$ and $Q \subseteq$ $C-P$. For any $x \in U$, we have

$$
\begin{aligned}
& \text { (1) } D_{P \cup Q}^{C D R+}(x)=D_{P}^{C D R+}(x) \cap D_{Q}^{C D R+}(x) \\
& \text { (2) } D_{P \cup Q}^{C D R-}(x)=D_{P}^{C D R-}(x) \cap D_{Q}^{C D R-}(x)
\end{aligned}
$$

Proof. (1) Assume $y \in D_{P}^{C D R+}(x) \cap D_{Q}^{C D R+}(x)$, namely $y \in D_{P}^{C D R+}(x)$ and $y \in D_{Q}^{C D R+}(x)$, which means $(x, y) \subseteq C D R(P)$ and $(x, y) \subseteq C D R(Q)$. It follows $y \in \bar{D}_{P \cup Q}^{C D R+}(x) . \quad D_{P \cup Q}^{C D R+}(x)=D_{P}^{C D R+}(x) \cap$ $D_{Q}^{C D R+}(x)$ holds.

(2) It is similar to (1).

By Lemma 1, Confidential dominating/dominated set of $x$ can be easily obtained when attribute set $Q$ is added into $P$.

Theorem 1 Let IODS $=(U, A, V, f), P \subset C$ and $Q \subseteq C-P$. For each $C l_{t}^{\geqslant}$, we have

(1) $P \cup Q\left(C l_{t}^{\geqslant}\right)=\underline{P}\left(C l_{t}^{\geqslant}\right) \cup T\left(C l_{t}^{\geqslant}\right)$,

where $T\left(C l_{t}^{\geqslant}\right)=\left\{x \mid l_{P \cup Q}\left(D_{P \cup Q}^{C D R+}(x)\right) \geqslant C l_{t}: x \in\right.$ $\left.B n_{P}\left(C l_{t}^{\geqslant}\right)\right\}$

(2) $\overline{P \cup Q}\left(C l_{t}^{\geqslant}\right)=\bar{P}\left(C l_{t}^{\geqslant}\right)-K\left(C l_{t}^{\geqslant}\right)$,

where $K\left(C l_{t}^{\geqslant}\right)=\left\{x \mid u_{P \cup Q}\left(D_{P \cup Q}^{C D R-}(x)\right)<C l_{t}: x \in\right.$ $\left.\bar{P}\left(C l_{t}^{\geqslant}\right)\right\}$

Proof. (1) $\underline{P \cup Q}\left(C l_{t}^{\geqslant}\right)=\left\{x \in U \mid D_{P \cup Q}^{C D R+}(x) \subseteq\right.$ $\left.C l_{t}^{\geqslant}\right\}$.

Since $D_{P \cup Q}^{C D R+}(x)=D_{P}^{C D R+}(x) \cap D_{Q}^{C D R+}(x)$, we have $\underline{P \cup Q}\left(C l_{t}^{\geqslant}\right)=\left\{x \in U \mid D_{P}^{C D R+}(x) \cap D_{Q}^{C D R+}(x) \subseteq\right.$ $\left.C l_{t}^{\geqslant}\right\}=\left\{x \in U \mid D_{P}^{C D R+}(x) \subseteq C l_{t}^{\geqslant}\right\} \cup\{x \in U-$ $\left.\underline{P}\left(C l_{t}^{\geqslant}\right) \mid D_{P \cup Q}^{C D R+}(x) \subseteq C l_{t}^{\geqslant}\right\}$. And $\{x \in U-$ $\left.\underline{P}\left(C l_{t}^{\geqslant}\right)\right\}=\left\{x \in\left(B n_{P}\left(C l_{t}^{\geqslant}\right) \cup\left(U-\bar{P}\left(C l_{t}^{\geqslant}\right)\right)\right\}\right.$, it follows $\left\{x \in U-\underline{P}\left(C l_{t}^{\geqslant}\right) \mid D_{P \cup Q}^{C D R+}(x) \subseteq C l_{t}^{\geqslant}\right\}=\{x \in$ $\left.B n_{P}\left(C l_{t}^{\geqslant}\right) \mid l_{P \cup Q}\left(D_{P \cup Q}^{C D R+}(x)\right) \geqslant C l_{t}\right\}$. Let $T\left(C l_{t}^{\geqslant}\right)=$ $\left\{x \mid l_{P \cup Q}\left(D_{P \cup Q}^{C D R+}(x)\right) \geqslant C l_{t}: x \in B n_{P}\left(C l_{t}^{\geqslant}\right)\right\}$. $\underline{P \cup Q}\left(C l_{t}^{\geqslant}\right)=\underline{P}\left(C l_{t}^{\geqslant}\right) \cup T\left(C l_{t}^{\geqslant}\right)$holds.

(2) Since $\overline{P \cup Q}\left(C l_{t}^{\geqslant}\right)=\cup_{x \in C l_{t}} D_{P \cup Q}^{C D R+}(x)=$ $\bigcup_{x \in C l_{t}}\left(D_{P}^{C D R+}(x) \cap D_{Q}^{C D R+}(x)\right)$

and $D_{P}^{C D R+}(x) \cap D_{Q}^{C D R+}(x)=D_{P}^{C D R+}(x)-(U-$ $\left.D_{P \cup Q}^{C D R+}(x)\right)$,

we have $\bigcup_{x \in C l_{t}}\left(D_{P}^{C D R+}(x) \cap D_{Q}^{C D R+}(x)\right)=$ $\cup_{x \in C l_{t}}\left(D_{P}^{C D R+}(x)-\left(U-D_{P \cup Q}^{C D R+}(x)\right)\right)$ $\left.=\bigcup_{x \in C l_{t}} D_{P}^{C D R+}(x)-\bigcup_{x \in C l_{t}}\left(U-D_{P \cup Q}^{C D R+}(x)\right)\right)$. According to Property 1.(4), we have $\overline{P \cup Q}\left(C l_{t}^{\geqslant}\right)=$ $\bar{P}\left(C l_{t}^{\geqslant}\right)-\left\{u_{P \cup Q}\left(D_{P \cup Q}^{C D R-}(x)\right)<C l_{t}: x \in \bar{P}\left(C l_{t}^{\geqslant}\right)\right\}$. Let $K\left(C l_{t}^{\geqslant}\right)=\left\{x \mid u_{P \cup Q}\left(D_{P \cup Q}^{C D R-}(x)\right)<C l_{t}: x \in\right.$ $\left.\bar{P}\left(C l_{t}^{\geqslant}\right)\right\}$.

$\overline{P \cup Q}\left(C l_{t}^{\geqslant}\right)=\bar{P}\left(C l_{t}^{\geqslant}\right)-K\left(C l_{t}^{\geqslant}\right)$holds.

Theorem 2 Let IODS $=(U, A, V, f), P \subset C$ and $Q \subseteq C-P$. For each $C l_{t}^{\leqslant}$, we have

$$
\begin{aligned}
& (1) \underline{P \cup Q}\left(C l_{t}^{\leqslant}\right)=\underline{P}\left(C l_{t}^{\leqslant}\right) \cup Y\left(C l_{t}^{\leqslant}\right), \\
& \text {where } Y\left(C l_{t}^{\leqslant}\right)=\left\{x \mid u_{P \cup Q}\left(D_{P \cup Q}^{C D R-}(x)\right) \leqslant C l_{t}: x \in\right.
\end{aligned}
$$
$\left.B n_{P}\left(C l_{t}^{\leqslant}\right)\right\}$

(2) $\overline{P \cup Q}\left(C l_{t}^{\leqslant}\right)=\bar{P}\left(C l_{t}^{\leqslant}\right)-Z\left(C l_{t}^{\leqslant}\right)$,

where $Z\left(C l_{t}^{\leqslant}\right)=\left\{x \mid l_{P \cup Q}\left(D_{P \cup Q}^{C D R+}(x)\right)>C l_{t}: x \in\right.$ $\left.\bar{P}\left(C l_{t}^{\leqslant}\right)\right\}$ 
Proof. It is similar to proof of Theorem 1.

Based on Theorems 1-2, an algorithm of updating approximation of CDRSA is proposed when new attributes are added into the IODS (See algorithm 1 IUA).

Example 2 (Continued from Example 1) Let $P=$ $\left\{a_{1}, a_{3}\right\}, Q=\left\{a_{2}\right\}, C=P \cup Q$.

$$
\begin{aligned}
& D_{P}^{C D R+}\left(x_{1}\right)=\left\{x_{1}, x_{10}\right\}, \\
& D_{P}^{C D R+}\left(x_{2}\right)=\left\{x_{1}, x_{2}, x_{10}\right\}, \\
& D_{P}^{C D R+}\left(x_{3}\right)=D_{P}^{C D R+}\left(x_{8}\right)=\left\{x_{1}, x_{3}, x_{8}, x_{10}\right\}, \\
& D_{P}^{C D R+}\left(x_{4}\right)=D_{P}^{C D R+}\left(x_{5}\right)=U-\left\{x_{7}\right\}, \\
& D_{P}^{C D R+}\left(x_{6}\right)=D_{P}^{C D R+}\left(x_{9}\right)=\left\{x_{1}, x_{2}, x_{3}, x_{6}, x_{8}, x_{9}, x_{10}\right\}, \\
& D_{P}^{C D R+}\left(x_{7}\right)=U . \\
& D_{Q}^{C D R+}\left(x_{1}\right)=\left\{x_{1}\right\}, \\
& D_{Q}^{C D R+}\left(x_{2}\right)=D_{Q}^{C D R+}\left(x_{4}\right)=D_{Q}^{C D R+}\left(x_{7}\right)= \\
& \left\{x_{1}, x_{2}, x_{4}, x_{7}, x_{10}\right\}, \\
& D_{Q}^{C D R+}\left(x_{3}\right)=D_{Q}^{C D R+}\left(x_{9}\right)=U, \\
& D_{Q}^{C D R+}\left(x_{5}\right)=D_{Q}^{C D R+}\left(x_{6}\right)=D_{Q}^{C D R+}\left(x_{8}\right)=U- \\
& \left\{x_{3}, x_{9}\right\} .
\end{aligned}
$$

Approximation of $C l_{t}^{\geqslant}$with respect to $P$ confidential dominating sets are computing as follows.

$\underline{P}\left(\mathrm{Cl}_{4}\right)=\emptyset, \underline{P}\left(C l_{3}^{\geqslant}\right)=\left\{x_{1}, x_{2}, x_{10}\right\}$,

$\underline{P}\left(C l_{2}^{\geqslant}\right)=\left\{x_{1}, x_{2}, x_{3}, x_{8}, x_{10}\right\}, \underline{P}\left(C l_{1}^{\geqslant}\right)=U$.

$\bar{P}\left(C l_{4}^{\geqslant}\right)=\left\{x_{1}, x_{10}\right\}, \bar{P}\left(C l_{3}^{\geqslant}\right)=\left\{x_{1}, x_{2}, x_{3}, x_{6}, x_{8}, x_{9}, x_{10}\right\}$, $\bar{P}\left(C l_{2}^{\geqslant}\right)=U, \bar{P}\left(C l_{1}^{\geqslant}\right)=U$.

According to Lemma $1, C=P \cup Q$ confidential dominating sets are easily calculated as follows. Take $x_{1}$ and $x_{4}$ for example.

$D_{C}^{C D R+}\left(x_{1}\right)=D_{P \cup Q}^{C D R+}\left(x_{1}\right)=D_{P}^{C D R+}\left(x_{1}\right) \cap$ $D_{Q}^{C D R+}\left(x_{1}\right)=\left\{x_{1}\right\}$.

$D_{P \cup Q}^{C D R+}\left(x_{4}\right)=D_{P}^{C D R+}\left(x_{4}\right) \cap D_{Q}^{C D R+}\left(x_{4}\right)=$ $\left\{x_{1}, x_{2}, x_{4}, x_{10}\right\}$.

Based on Theorem 2, approximations of $\mathrm{Cl}_{t}^{\geqslant}$ with respect to $P \cup Q$ confidential dominating sets are computing as follows.

$$
T\left(C l_{4}^{\geqslant}\right)=\left\{x \mid l_{P \cup Q}\left(D_{P \cup Q}^{C D R+}(x)\right) \geqslant C l_{4}: x \in\right.
$$

$\left.B n_{P}\left(C l_{4}^{\geqslant}\right)\right\}=\left\{x_{1}\right\}$,

$T\left(C l_{3}^{\geqslant}\right)=\emptyset, T\left(C l_{2}^{\geqslant}\right)=\left\{x_{4}, x_{7}\right\}, T\left(C l_{1}^{\geqslant}\right)=\emptyset$.

$K\left(C l_{4}^{\geqslant}\right)=\left\{x \mid u_{P \cup Q}\left(D_{P \cup Q}^{C D R-}(x)\right)<C l_{4}: x \in\right.$ $\left.\bar{P}\left(C l_{4}^{\geqslant}\right)\right\}=\left\{x_{10}\right\}$,

$\mathrm{K}\left(\mathrm{Cl} l_{3}^{\geqslant}\right)=K\left(C l_{2}^{\geqslant}\right)=K\left(C l_{1}^{\geqslant}\right)=\emptyset$.

$$
\underline{P \cup Q}\left(C l_{4}^{\geqslant}\right)=\underline{P}\left(C l_{4}^{\geqslant}\right) \cup T\left(C l_{4}^{\geqslant}\right)=\left\{x_{1}\right\},
$$

$P \cup \overline{Q(C l} \geqslant)=\left\{x_{1}, x_{2}, x_{10}\right\}, \quad P \cup Q\left(C l_{2}^{\geqslant}\right)=$ $\left\{x_{1}, x_{2}, x_{3}, x_{4}, x_{7}, x_{8}, x_{10}\right\}, P \cup Q\left(C l_{1}^{\geqslant}\right)=U$.

$\overline{P \cup Q}\left(C l_{4}^{\geqslant}\right)=\bar{P}\left(\mathrm{Cl}_{4}^{\geqslant}\right)-K\left(C l_{4}^{\geqslant}\right)=\left\{x_{1}\right\}$, $\overline{P \cup Q}\left(C l_{3}^{\geqslant}\right)=\left\{x_{1}, x_{2}, x_{3}, x_{6}, x_{8}, x_{9}, x_{10}\right\} . \bar{P}\left(C l_{2}^{\geqslant}\right)=$ $\bar{P}\left(C l_{1}^{\geqslant}\right)=U$.

\subsection{Incremental updating approximations when attributes deleted}

Lemma 2 Let IODS $=(U, A, V, f), P \subset C$ and $Q \subset$ $P \subseteq C$. For any $x \in U$, we have

(1) $D_{P-Q}^{C D R+}(x)=D_{P}^{C D R+}(x) \cup \mathrm{A}_{Q}^{\geqslant}(x)$,

where $\mathrm{A}_{Q}^{\geqslant}(x)=\left\{y \in U-D_{P}^{C D R+}(x) \mid(U-\right.$ $\left.\left.D_{P}^{C D R+}(x)\right) \wedge D_{P-Q}^{C D R+}(x)\right\}$.

(2) $D_{P-Q}^{C D R-}(x)=D_{P}^{C D R-}(x) \cup \mathrm{A}_{Q}^{\leqslant}(x)$,

where $\mathrm{A}_{Q}^{\leqslant}(x)=\left\{y \in U-D_{P}^{C D R-}(x) \mid(U-\right.$ $\left.\left.D_{P}^{C D R-}(x)\right) \wedge D_{P-Q}^{C D R-}(x)\right\}$.

Proof. (1) Proof by contradiction. Assume $y \in$ $D_{P-Q}^{C D R+}(x)$ satisfies $y \in U-D_{P}^{C D R+}(x)$ and $y \notin \mathrm{A}_{Q}^{\geqslant}(x)$, namely, $y \notin\left\{U-D_{P}^{C D R+}(x) \wedge D_{P-Q}^{C D R+}(x)\right\}$, then $y \in$ $D_{P}^{C D R+}(x)$ or $y \in U-D_{P-Q}^{C D R+}(x)$. (a) $y \in D_{P}^{C D R+}(x)$ is in contradiction with the assumption $y \in U-$ $D_{P}^{C D R+}(x)$; (b) $y \in U-D_{P-Q}^{C D R+}(x)$, we have $(x, y) \subseteq$ $U-D_{P-Q}^{C D R+}(x)$ which means $y \notin D_{P-Q}^{C D R+}(x)$. Contradiction. $D_{P-Q}^{C D R+}(x)=D_{P}^{C D R+}(x) \cup \mathrm{A}_{Q}^{\geqslant}(x)$ holds.

(2) It is similar to Lemma 2(1).

Theorem 3 Let IODS $=(U, A, V, f), P \subset C$ and $Q \subset P \subseteq C$. For any $x \in U$, we have

(1) $\underline{P-Q}\left(C l_{t}^{\geqslant}\right)=\underline{P}\left(C l_{t}^{\geqslant}\right)-W\left(C l_{t}^{\geqslant}\right)$,

where $W\left(C l_{t}^{\geqslant}\right)=\left\{x \mid l_{P-Q}\left(\mathrm{~A}_{Q}^{\geqslant}(x)\right)<C l_{t}, x \in\right.$ $\left.\underline{P}\left(C l_{t}^{\geqslant}\right)\right\}$

(2) $\overline{P-Q}\left(C l_{t}^{\geqslant}\right)=\bar{P}\left(C l_{t}\right) \cup R\left(C l_{t}^{\geqslant}\right)$, where $R\left(C l_{t}^{\geqslant}\right)=\left\{\mathrm{A}_{Q}^{\geqslant}(x): x \in \bar{P}\left(C l_{t}^{\geqslant}\right)\right\}$

Proof. (1) $P-Q\left(C l_{t}^{\geqslant}\right)=\left\{x \in U \mid D_{P-Q}^{C D R+}(x) \subseteq\right.$ $\left.C l_{t}^{\geqslant}\right\}=\underline{P}\left(C l_{t}^{\geqslant}\right)-\left\{x \in \underline{P}\left(C l_{t}^{\geqslant}\right) \mid D_{P-Q}^{C D R+}(x) \nsubseteq C l_{t}^{\geqslant}\right\}$. Let $W\left(C l_{t}^{\geqslant}\right)=\left\{x \in \underline{P}\left(C l_{t}^{\geqslant}\right) \mid D_{P-Q}^{C D R+}(x) \nsubseteq C l_{t}^{\geqslant}\right\}=$ $\left\{x \mid l_{P-Q}\left(\mathrm{~A}_{Q}^{\geqslant}(x)\right)<C l_{t}, x \in \underline{P}\left(C l_{t}^{\geqslant}\right)\right\}$.

$P-Q\left(C l_{t}^{\geqslant}\right)=\underline{P}\left(C l_{t}^{\geqslant}\right)-W\left(C l_{t}^{\geqslant}\right)$holds.

(2) $\overline{P-Q}\left(C l_{t}^{\geqslant}\right)=\bigcup_{x \in C l_{t}^{\geqslant}} D_{P-Q}^{C D R+}(x)=\bar{P}\left(C l_{t}^{\geqslant}\right) \cup$ $\left\{\mathrm{A}_{Q}^{\geqslant}(x): x \in \bar{P}\left(C l_{t}^{\geqslant}\right)\right\}$. Let $R\left(C l_{t}^{\geqslant}\right)=\left\{\mathrm{A}_{Q}^{\geqslant}(x): x \in\right.$ $\left.\bar{P}\left(C l_{t}^{\geqslant}\right)\right\}$.

$\overline{P-Q}\left(C l_{t}^{\geqslant}\right)=\bar{P}\left(C l_{t}^{\geqslant}\right) \cup R\left(C l_{t}^{\geqslant}\right)$holds. 


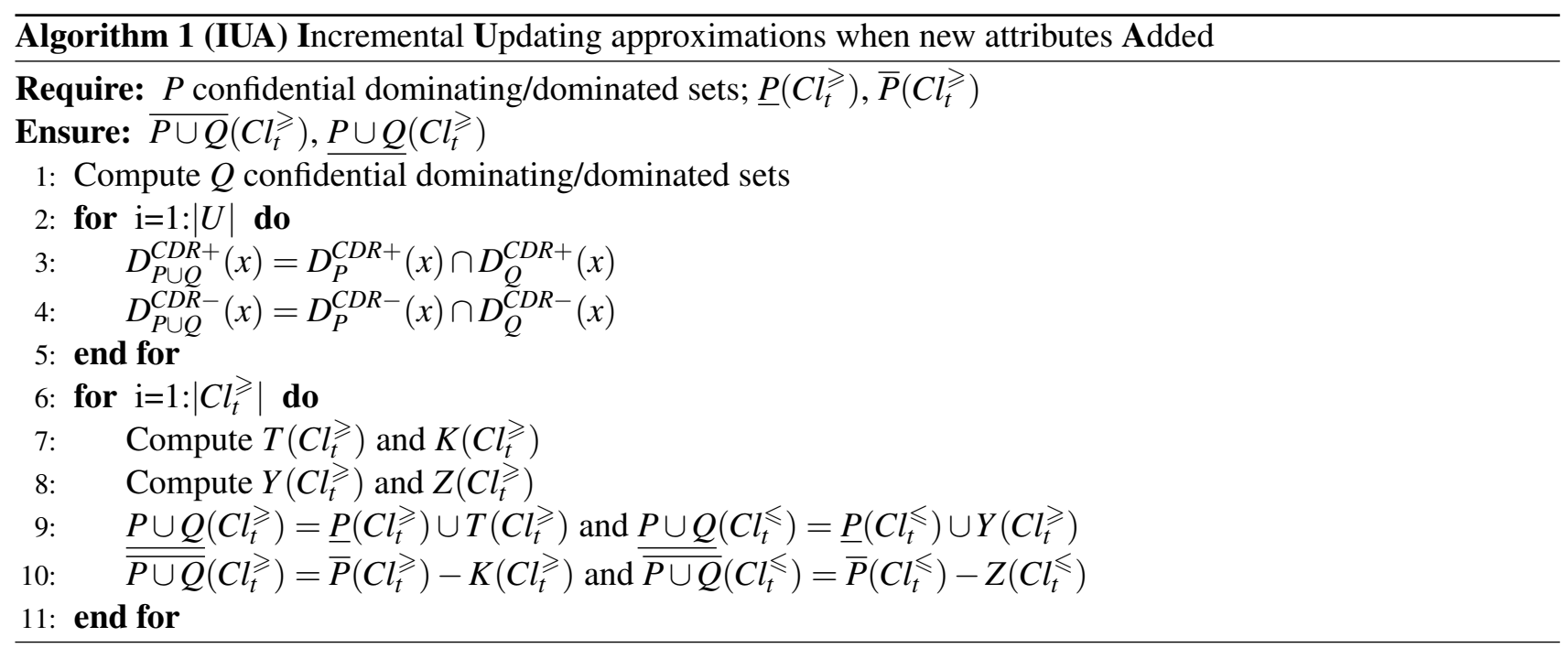

Theorem 4 Let IODS $=(U, A, V, f), P \subset C$ and $Q \subset P \subseteq C$. For any $x \in U$, we have

(1) $P-Q\left(C l_{t}^{\leqslant}\right)=\underline{P}\left(C l_{t}^{\leqslant}\right)-S\left(C l_{t}^{\leqslant}\right)$, where $S\left(C l_{t}^{\leqslant}\right)=\left\{x \mid u_{P-Q}\left(\mathrm{~A}_{Q}^{\leqslant}(x)\right)>C l_{t}, x \in\right.$ $\left.\underline{P}\left(C l_{t}^{\leqslant}\right)\right\}$

(2) $\overline{P-Q}\left(C l_{t}^{\leqslant}\right)=\bar{P}\left(C l_{t}^{\leqslant}\right) \cup V\left(C l_{t}^{\leqslant}\right)$, where $V\left(C l_{t}^{\leqslant}\right)=\left\{\mathrm{A}_{Q}^{\leqslant}(x): x \in \bar{P}\left(C l_{t}^{\leqslant}\right)\right\}$

Proof. It is similar to proof of Theorem 3.

Based on Theorems 3-4, an algorithm is developed for updating approximations of CDRSA when some attributes are deleted from the IODS (see Algorithm 2 IUD).

Example 3 (Continued from Example 1) Let $x \in U$, $P=C-Q$.

According to Lemma 2, for any $x, \mathrm{~A}_{Q}^{\geqslant}(x)$ is easily calculated as follows.

$\mathrm{A}_{Q}^{\geqslant}\left(x_{1}\right)=\left\{x_{10}\right\}, \mathrm{A}_{Q}^{\geqslant}\left(x_{2}\right)=\mathrm{A}_{Q}^{\geqslant}\left(x_{3}\right)=\mathrm{A}_{Q}^{\geqslant}\left(x_{9}\right)=$ $\mathrm{A}_{Q}^{\geqslant}\left(x_{10}\right)=\emptyset$,

$\mathrm{A}_{Q}^{\geqslant}\left(x_{4}\right)=\left\{x_{3}, x_{5}, x_{6}, x_{8}, x_{9}, x_{10}\right\}, \quad \mathrm{A}_{Q}^{\geqslant}\left(x_{5}\right)=$ $\mathrm{A}_{Q}^{\geqslant}\left(x_{6}\right)=\left\{x_{3}, x_{9}\right\}$,

$\mathrm{A}_{Q}^{\geqslant}\left(x_{7}\right)=\left\{x_{3}, x_{5}, x_{6}, x_{8}, x_{9}\right\}, \mathrm{A}_{Q}^{\geqslant}\left(x_{8}\right)=\left\{x_{3}\right\}$

Approximation of $C l_{t}^{\geqslant}$with respect to $P=C-Q$ confidential dominating sets are computing as follows.

$W\left(C l_{4}^{\geqslant}\right)=\left\{x \mid l_{C-Q}\left(\mathrm{~A}_{Q}^{\geqslant}(x)\right)<C l_{4}, x \in\right.$ $\left.\underline{P}\left(C l_{4}^{\geqslant}\right)\right\}=\left\{x_{1}\right\}, W\left(C l_{3}^{\geqslant}\right)=\emptyset, W\left(C l_{2}^{\geqslant}\right)=\left\{x_{4}, x_{7}\right\}$,
$W\left(C l_{1}^{\geqslant}\right)=\emptyset$.

$R\left(C l_{4}^{\geqslant}\right)=\left\{\mathrm{A}_{Q}^{\geqslant}(x): x \in \bar{P}\left(C l_{4}^{\geqslant}\right)\right\}=$ $\left\{x_{10}\right\}, \quad R\left(C l_{3}^{\geqslant}\right)=\left\{x_{3}, x_{9}, x_{10}\right\}, \quad R\left(C l_{2}\right)=$ $\left\{x_{3}, x_{5}, x_{6}, x_{8}, x_{9}, x_{10}\right\}, R\left(C l_{1}^{\geqslant}\right)=\left\{x_{3}, x_{5}, x_{6}, x_{8}, x_{9}, x_{10}\right\}$. $\underline{P}=C-Q\left(C l_{4}^{\geqslant}\right)=\underline{C}-W\left(C l_{4}^{\geqslant}\right)=\emptyset$, $\underline{C-Q}\left(C l_{3}^{\geqslant}\right)=\left\{x_{1}, x_{2}, x_{10}\right\}, \quad \underline{C-Q}\left(C l_{2}^{\geqslant}\right)=$ $\left.\overline{\left\{x_{1}, x_{2}\right.}, x_{3}, x_{8}, x_{10}\right\}, \underline{C-Q}\left(C l_{1}^{\geqslant}\right)=U$

$\bar{P}\left(\mathrm{Cl}_{4}^{\geqslant}\right)=\underline{\mathrm{C}}-\overline{\mathrm{Q}}\left(\mathrm{Cl} l_{4}^{\geqslant}\right)=\underline{\mathrm{C}}\left(\mathrm{Cl}_{4}^{\geqslant}\right) \cup \mathrm{R}\left(\mathrm{Cl}_{4}\right)=$ $\left\{x_{1}, x_{10}\right\}$,

$\bar{P}\left(C l_{3}\right)=\left\{x_{1}, x_{2}, x_{3}, x_{6}, x_{8}, x_{9}, x_{10}\right\}, \bar{P}\left(C l_{2}^{\geqslant}\right)=U$, $\bar{P}\left(C l_{1}^{\geqslant}\right)=U$

\section{Incremental updating approximations when subsets of objects are merged}

In this section, incremental updating approximations of CDRSA is discussed when subsets of objects are merged into IODS.

Assume the universe of $I O D S=(U, A, V, f)$ is composed by $m$ subsets of objects, where $U=$ $\bigcup_{i=1}^{m} U_{i}, i=1,2, \cdots, m$.

Definition 7 Let $U_{k} \subset U, k=1,2, \cdots, m . C l_{t}^{\geqslant k}$ and $C l_{t}^{\leqslant k}$ in subset $U_{k}$ are defined as follows.

$$
\begin{aligned}
& C l_{t}^{\geqslant k}=\left\{x \in U_{k} \mid f(x, d) \geqslant C l_{t}\right\} \\
& C l_{t}^{\leqslant k}=\left\{x \in U_{k} \mid f(x, d) \leqslant C l_{t}\right\}
\end{aligned}
$$




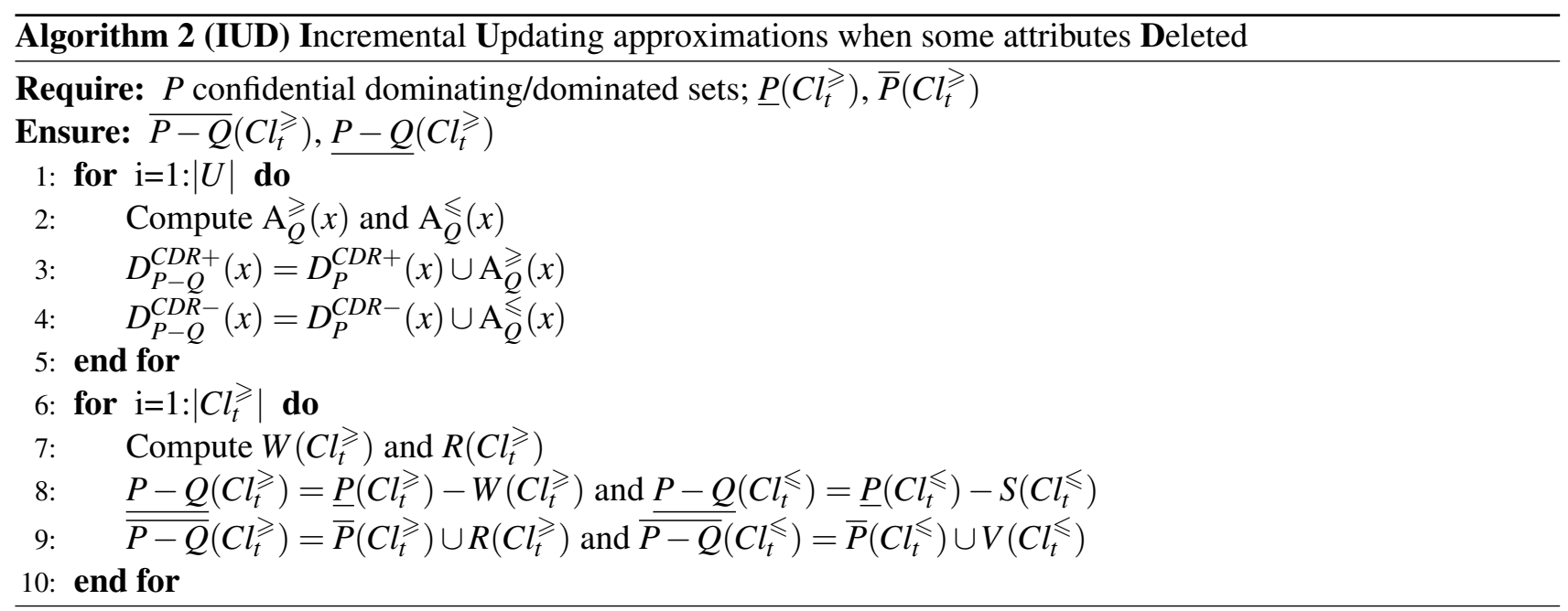

Definition 8 Let $U_{i} \subset U, i=1,2, \cdots, m$. The $P$ confidential dominating/dominated sets of the object $x$ related to $U_{i}$ are defined as follows.

$$
\begin{aligned}
& D_{P}^{C D R+}(x)^{i}=\left\{y \in U_{i} \mid y D_{P}^{C D R} x\right\} \\
& D_{P}^{C D R-}(x)^{i}=\left\{y \in U_{i} \mid x D_{P}^{C D R} y\right\}
\end{aligned}
$$

Definition 9 Let $U_{k} \subset U, i, k=1,2, \cdots, m$. Lower and upper approximations of $\mathrm{Cl}_{t}^{\geqslant k}$ related to $U_{i}$ are defined as follows.

$$
\begin{gathered}
\underline{P}\left(C l_{t}^{\geqslant k}\right)^{i}=\left\{x \in U_{i} \mid D_{P}^{C D R+}(x)^{i} \subseteq C l_{t}^{\geqslant k}\right\} \\
\bar{P}\left(C l_{t}^{\geqslant k}\right)^{i}=\bigcup_{x \in C l_{t}^{\geqslant k}} D_{P}^{C D R+}(x)^{i}=\left\{x \in U_{i} \mid D_{P}^{C D R-}(x)^{i} \cap C l_{t}^{\geqslant k} \neq \emptyset\right\}
\end{gathered}
$$

Theorem 6 Let $x \in U, i=1,2, \cdots, m$, we have

(1) $D_{P}^{C D R+}(x)=\bigcup_{i=1}^{m} D_{P}^{C D R+}(x)^{i}$

(2) $D_{p}^{C D R-}(x)=\bigcup_{i=1}^{m} D_{P}^{C D R-}(x)^{i}$

Proof. (1) Since $D_{P}^{C D R+}(x)=\left\{x \in U \mid y D_{P}^{C D R} x\right\}=$ $\left\{x \in \bigcup_{i=1}^{m} U_{i} \mid y D_{P}^{C D R} x\right\}=\bigcup_{i=1}^{m}\left\{x \in U_{i} \mid y D_{P}^{C D R} x\right\}=$ $\bigcup_{i=1}^{m} D_{P}^{C D R+}(x)^{i}, D_{P}^{C D R+}(x)=\bigcup_{i=1}^{m} D_{P}^{C D R+}(x)^{i}$ holds.

(2) It is similar to proof Theorem 6(1).

Theorem 7 Given $t=1,2, \cdots, n, i=1,2, \cdots, m$, the following items hold.

(1) $\underline{P}\left(C l_{t}^{\geqslant}\right)=\bigcup_{i=1}^{m}\left(\bigcap_{k=1}^{m} \underline{P}\left(C l_{t}^{\geqslant k}\right)^{i}\right)$

(2) $\bar{P}\left(C l_{t}^{\geqslant}\right)=\bigcup_{i=1}^{m} \bigcup_{k=1}^{m} \bar{P}\left(C l_{t}^{\geqslant k}\right)^{i}$

Proof. (1) Since $\underline{P}\left(C l_{t}^{\geqslant}\right)=\left\{x \in U \mid D_{p}^{C D R+}(x) \subseteq\right.$ $\left.C l_{t}^{\geqslant}\right\}=$

Similarly, lower and upper approximations of $C l_{t}^{\leqslant k}$ related to $U_{i}$ are defined as follows.

$$
\underline{P}\left(C l_{t}^{\leqslant k}\right)^{i}=\left\{x \in U_{i} \mid D_{P}^{C D R-}(x)^{i} \subseteq C l_{t}^{\leqslant k}\right\}
$$

$\left\{x \in \bigcup_{i=1}^{m} U_{i} \mid \bigcup_{k=1}^{m} D_{p}^{C D R+}(x)^{k} \subseteq \bigcup_{k=1}^{m} C l_{t}^{\geqslant k}\right\}=$

$\bigcup_{i=1}^{m}\left\{x \in U_{i} \mid D_{p}^{C D R+}(x)^{1} \subseteq C l_{t}^{\geqslant 1} \wedge \cdots \wedge D_{p}^{C D R+}(x)^{m} \subseteq\right.$ $\bar{P}\left(C l_{t}^{\leqslant k}\right)^{i}=\bigcup D_{P}^{C D R-}(x)^{i}=\left\{x \in U_{i} \mid D_{P}^{C D R+}(x)^{i} \cap C l_{t}^{\leqslant k} \neq \bigcup \bigcup_{\}_{i=1}^{m}} l_{t}^{\geqslant m}\right\}=$

$$
\text { Let } i=1,2, \cdots, m, t=1,2, \cdots, n \text {, we }
$$

Theorem 5 Let $i=1,2, \cdots, m, t=1,2, \cdots, n$, we have

(1) $C l_{t}^{\geqslant}=\bigcup_{i=1}^{m} C l_{t}^{\geqslant i}$

(2) $C l_{t}^{\leqslant}=\bigcup_{i=1}^{m} C l_{t}^{\leqslant i}$

Proof. (1) Since $C l_{t}^{\geqslant}=\left\{x \in U \mid f(x, D) \geqslant C l_{t}\right\}=$ $\left\{x \in \bigcup_{i=1}^{m} U_{i} \mid f(x, D) \geqslant C l_{t}\right\}=\bigcup_{i=1}^{m}\{x \in$ $\left.U_{i} \mid f(x, D) \geqslant C l_{t}\right\}=\bigcup_{i=1}^{m} C l_{t}^{\geqslant i}, C l_{i}^{\geqslant}=\bigcup_{k=1}^{m} C l_{t}^{\geqslant i}$ holds.

(2) It is similar to proof Theorem 5(1). $\bigcup_{i=1}^{m}\left(\bigcap_{k=1}^{m} \underline{P}\left(C l_{t}^{\geqslant k}\right)^{i}\right)$,

$\underline{P}\left(C l_{t}^{\geqslant}\right)=\bigcup_{i=1}^{m}\left(\bigcap_{k=1}^{m} \underline{P}\left(C l_{t}^{\geqslant k}\right)^{i}\right)$ holds.

(2) Since $\bar{P}\left(C l_{t}^{\geqslant}\right)=\left\{x \in U \mid D_{p}^{C D R-}(x) \cap C l_{t}^{\geqslant} \neq\right.$ $\emptyset\}=$

$\left\{x \in \bigcup_{i=1}^{m} U_{i} \mid \bigcup_{k=1}^{m} D_{p}^{C D R-}(x)^{k} \cap \bigcup_{k=1}^{m} C l_{t}^{\geqslant k} \neq \emptyset\right\}=$ $\bigcup_{i=1}^{m}\left\{x \in U_{i} \mid D_{p}^{C D R-}(x)^{1} \cap C l_{t}^{\geqslant 1} \neq \emptyset \wedge \cdots \wedge\right.$ $\left.D_{p}^{C D R-}(x)^{m} \cap C l_{t}^{\geqslant m} \neq \emptyset\right\}=\bigcup_{i=1}^{m}\left(\bigcup_{k=1}^{m}\{x \in\right.$ $\left.\left.U_{i} \mid D_{p}^{C D R-}(x)^{k} \cap C l_{t}^{\geqslant k} \neq \emptyset\right\}\right)=\bigcup_{i=1}^{m} \bigcup_{k=1}^{m} \bar{P}\left(C l_{t}^{\geqslant k}\right)^{i}$, $\bar{P}\left(C l_{t}^{\geqslant}\right)=\bigcup_{i=1}^{m} \bigcup_{k=1}^{m} \bar{P}\left(C l_{t}^{\geqslant k}\right)^{i}$ holds. 
Theorem 8 Given $t=1,2, \cdots, n, i=1,2, \cdots, m$, the following items hold.
(1) $\underline{P}\left(C l_{t}^{\leqslant}\right)=\cup_{i=1}^{m}\left(\cap_{k=1}^{m} \underline{P}\left(C l_{t}^{\leqslant k}\right)^{i}\right)$
(2) $\bar{P}\left(C l_{t}^{\leqslant}\right)=\cup_{i=1}^{m} \cup_{k=1}^{m} \bar{P}\left(C l_{t}^{\leqslant k}\right)^{i}$

Proof. It is similar to proof of Theorem 7.

Based on Theorems 5-8, an algorithm is designed for updating approximations of CDRSA when subsets of objects are merged IODS (see Algorithm 3 IUM).

Example 4 (Continued from Example 1) Let $U=$ $U_{1} \cup U_{2}$, where $U_{1}=\left\{x_{1}, x_{2}, \cdots, x_{7}\right\}$ and $U_{2}=$ $\left\{x_{8}, x_{9}, x_{10}\right\}$.

According to Definition, $k=1,2 . \mathrm{Cl}_{t}^{\geqslant k}$ in subset $U_{k}$ are calculated as follows.

$$
C l_{4}^{\geqslant 1}=\left\{x_{1}\right\}, C l_{3}^{\geqslant 1}=\left\{x_{1}, x_{2}, x_{3}\right\}, \quad C l_{2}^{\geqslant 1}=
$$
$\left\{x_{1}, x_{2}, x_{3}, x_{4}, x_{5}, x_{7}\right\}, C l_{1}^{\geqslant 1}=U_{1}$. $U_{2}$.

$C l_{3}^{\geqslant 2}=\left\{x_{9}, x_{10}\right\}, C l_{2}^{\geqslant 2}=\left\{x_{8}, x_{9}, x_{10}\right\}, C l_{1}^{\geqslant 2}=$

The $C$ confidential dominating sets of the object $x$ related to $U_{i}(i=1,2)$ are calculated as follows.

$D_{C}^{C D R+}\left(x_{1}\right)^{1}=\left\{x_{1}\right\}, D_{C}^{C D R+}\left(x_{2}\right)^{1}=\left\{x_{1}, x_{2}\right\}$, $D_{C}^{C D R+}\left(x_{3}\right)^{1}=\left\{x_{1}, x_{3}\right\}, D_{C}^{C D R+}\left(x_{4}\right)^{1}=\left\{x_{1}, x_{2}, x_{4}\right\}$, $D_{C}^{C D R+}\left(x_{5}\right)^{1}=\left\{x_{1}, x_{2}, x_{4}, x_{5}, x_{6}\right\}$,

$D_{C}^{C D R+}\left(x_{6}\right)^{1}=\left\{x_{1}, x_{2}, x_{6}\right\}, \quad D_{C}^{C D R+}\left(x_{7}\right)^{1}=$ $\left\{x_{1}, x_{2}, x_{4}, x_{7}\right\}$.

$D_{C}^{C D R+}\left(x_{1}\right)^{2}=\emptyset, \quad D_{C}^{C D R+}\left(x_{2}\right)^{2}=\left\{x_{10}\right\}$, $D_{C}^{C D R+}\left(x_{3}\right)^{2}=\left\{x_{8}, x_{10}\right\}$,

$D_{C}^{C D R+}\left(x_{4}\right)^{2}=\left\{x_{10}\right\}, \quad D_{C}^{C D R+}\left(x_{5}\right)^{2}=\left\{x_{8}, x_{10}\right\}$, $D_{C}^{C D R+}\left(x_{6}\right)^{2}=\left\{x_{8}, x_{10}\right\}$,

$D_{C}^{C D R+}\left(x_{7}\right)^{2}=\left\{x_{10}\right\}$.

$D_{C}^{C D R+}\left(x_{8}\right)^{1}=\left\{x_{1}\right\}, D_{C}^{C D R+}\left(x_{9}\right)^{1}=\left\{x_{1}, x_{2}, x_{3}, x_{6}\right\}$, $D_{C}^{C D R+}\left(x_{10}\right)^{1}=\left\{x_{1}\right\}$.

$D_{C}^{C D R+}\left(x_{8}\right)^{2}=\left\{x_{8}, x_{10}\right\}, \quad D_{C}^{C D R+}\left(x_{9}\right)^{2}=$ $\left\{x_{8}, x_{9}, x_{10}\right\}$,

$D_{C}^{C D R+}\left(x_{10}\right)^{2}=\left\{x_{10}\right\}$.

Lower approximations of $C l_{t}^{\geqslant k}$ related to $U_{i}$, where $i, k=1,2$, are computed as follows.

$$
\underline{C}\left(C l_{4}^{\geqslant 1}\right)^{1}=\left\{x_{1}\right\}, \underline{C}\left(C l_{4}^{\geqslant 2}\right)^{1}=\left\{x_{1}\right\},
$$
$\underline{C}\left(C l_{3}^{\geqslant 1}\right)^{1}=\left\{x_{1}, x_{2}, x_{3}\right\}, \underline{C}\left(C l_{3}^{\geqslant 2}\right)^{1}=\left\{x_{1}, x_{2}, x_{4}, x_{7}\right\}$, $\underline{C}\left(C l_{2}^{\geqslant 1}\right)^{1}=\left\{x_{1}, x_{2}, x_{3}, x_{4}, x_{7}\right\}, \underline{C}\left(C l_{2}^{\geqslant 2}\right)^{1}=U_{1}$.

$\underline{C}\left(C l_{4}^{\geqslant 1}\right)^{2}=\emptyset, \underline{C}\left(C l_{4}^{\geqslant 2}\right)^{2}=\left\{x_{8}, x_{10}\right\}$,

$\underline{C}\left(l_{l}^{\geqslant 1}\right)^{2}=\left\{x_{8}, x_{10}\right\}, \quad \underline{C}\left(C l_{3}^{\geqslant 2}\right)^{2}=\left\{x_{10}\right\}$,
$\underline{C}\left(C l_{2}^{\geqslant 1}\right)^{2}=\left\{x_{8}, x_{10}\right\}$,

$\underline{C}\left(\mathrm{Cl}_{2}^{\geqslant 2}\right)^{2}=U_{2}$.

Upper approximations of $\mathrm{Cl}_{t}^{\geqslant k}$ related to $U_{i}$, where $i, k=1,2$, are computed as follows.

$\bar{C}\left(\mathrm{Cl}_{4}^{\geqslant 1}\right)^{1}=\left\{x_{1}\right\}, \quad \bar{C}\left(\mathrm{Cl}_{3}^{\geqslant 1}\right)^{1}=\left\{x_{1}, x_{2}, x_{3}\right\}$, $\bar{C}\left(C l_{2}^{\geqslant 1}\right)^{1}=\bar{C}\left(C l_{1}^{\geqslant 1}\right)^{1}=U_{1}$

$\bar{C}\left(C l_{4}^{\geqslant 1}\right)^{2}=\emptyset, \quad \bar{C}\left(C l_{3}^{\geqslant 1}\right)^{2}=\bar{C}\left(C l_{2}^{\geqslant 1}\right)^{2}=$ $\bar{C}\left(C l_{1}^{\geqslant 1}\right)^{2}=\left\{x_{1}, x_{2}, x_{3}, x_{6}\right\}$.

$\bar{C}\left(\mathrm{Cl}_{3}^{\geqslant 2}\right)^{1}=\bar{C}\left(\mathrm{Cl}_{2}^{\geqslant 2}\right)^{1}=\left\{x_{8}, x_{9}, x_{10}\right\}$.

$\bar{C}\left(C l_{3}^{\geqslant 2}\right)^{2}=\bar{C}\left(C l_{2}^{\geqslant 2}\right)^{2}=\left\{x_{8}, x_{9}, x_{10}\right\}$.

Approximations of $C l_{t}^{\geqslant}$when $U_{1}$ and $U_{2}$ merged are updated as follows.

$\underline{C}\left(C l_{t}^{\geqslant}\right)=\bigcup_{i=1}^{2}\left(\bigcap_{k=1}^{2} \underline{C}\left(C l_{t}^{\geqslant k}\right)^{i}\right)=\left\{x_{1}\right\}$, $\underline{C}\left(C_{3}\right)=\left\{x_{1}, x_{2}, x_{10}\right\}$,

$\underline{C}\left(C l_{2}^{\geqslant}\right)=\left\{x_{1}, x_{2}, x_{3}, x_{4}, x_{7}, x_{8}, x_{10}\right\}, \underline{C}\left(C l_{1}^{\geqslant}\right)=U_{1} \cup$ $U_{2}$.

Approximations of $C l_{t}^{\geqslant}$when $U_{1}$ and $U_{2}$ merged are updated as follows.

$\bar{C}\left(C l_{4}^{\geqslant}\right)=\bigcup_{i=1}^{2} \bigcup_{k=1}^{2} \bar{C}\left(C l_{4}^{\geqslant k}\right)^{i}=\left\{x_{1}\right\}, \bar{C}\left(C l_{3}^{\geqslant}\right)=$ $\left\{x_{1}, x_{2}, x_{3}, x_{6}, x_{8}, x_{9}, x_{10}\right\}, \bar{C}\left(C l_{2}^{\geqslant}\right)=U, \bar{C}\left(C l_{1}^{\geqslant}\right)=U$.

\section{Expriment}

Some experiments are conducted to evaluate the performance of the incremental approaches. Six UCI data sets are selected from UCI Machine Learning Repository ${ }^{50}$, described in Table 2, as benchmark for performance test. The incremental algorithms and non-incremental algorithm are developed by Matlab 2014Ra on Macbook Pro 2015 with OS X EI Capitan on Intel Core i5 $2.7 \mathrm{GHz}$ and $16 \mathrm{G}$ memory.

There are two classes of the experiments: experiments on updating approximations of CDRSA under the variation of attributes and experiments on updating approximations when two subsets are combined.

Table 2. Data sets description

\begin{tabular}{clccc}
\hline ID & Data set & $|U|$ & $|C|$ & class \\
\hline 1 & heart-disease(cleveland) & 303 & 13 & 5 \\
2 & lonosphere & 351 & 34 & 2 \\
3 & arrhythmia & 452 & 279 & 12 \\
4 & winequality(white) & 4898 & 11 & 7 \\
5 & statlog(Landsat Satellite) & 6435 & 36 & 6 \\
6 & mushroom & 7910 & 22 & 7 \\
\hline
\end{tabular}




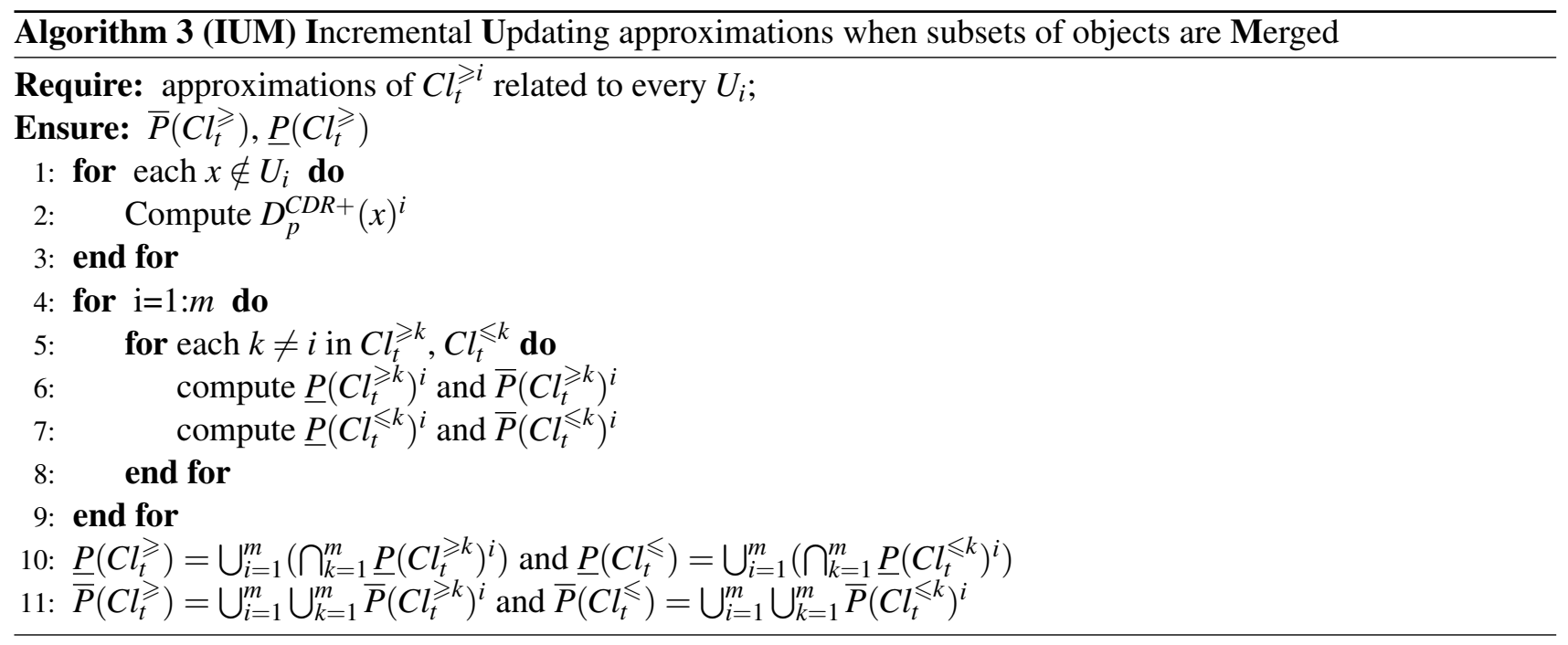

\subsection{The variation of attributes}

For this case, a comparison of incremental updating approximations and non-incremental computing approximations is investigated to evaluate the performance of updating approximations of CDRSA under the variation of the attribute set. We make two groups of experiments on each data sets when some attributes are deleted and added correspondingly.

In the first group of experiments, some attributes are randomly selected from the condition attribute $C$ to constitute a deleted attribute set $Q$. The size of $Q$ is from $10 \%$ to $50 \%$ in percentage in $C$, where the step length is $10 \%$. The remaining attributes are $P=C-Q$. Time consuming is recorded to compare the incremental approach of updating approximations IUD and non-incremental method of computing approximations with respect to $P$ when attribute set $Q$ is deleted from $C$.

In the second group of experiments, the deleted attribute set $Q$ are added back into $P$. Thus, we have $C=P \cup Q$. Similarly, computational time of incremental approach IUA is compared with nonincremental method when attribute set $Q$ is added into $P$.

The experimental results of the six data sets are shown in Figure 1. In each of sub-figures, the $x$ coordinate pertains to percentage of the size of $Q$ in $C$ from $10 \%$ to $50 \%$, and $y$-coordinate pertains the time consuming. The 'non-incr-add' denotes non- incremental method of computing approximations with respect to $C$, while the 'non-incr-del' denotes non-incremental computing approximations with respect to $P$.

From the Figure 1, the incremental approaches IUA and IUD are always faster than nonincremental approach 'non-incr-add' and 'non-incrdel' respectively. Furthermore, IUA is always effective than IUD because calculation of the confidential dominating/dominated sets in IUA is faster.

The experimental results of data sets arrhythmia and statlog (Landsat Satellite) with large condition attributes, as in Figure 1 (c) and Figure 1(e), show that the trend of time consuming of both IUD and 'non-incr-del' goes down when the cardinality of deleted attribute set increases.

In addition, from the results of winequality(white), statlog (Landsat Satellite) and mushroom with more objects, the trend of time consuming of IUA goes down when the cardinality of added attribute set increases. Intuitively, the trend of time consuming should go up when the cardinality of added attribute set increases. However, the boundary of remain attributes may decrease after the cardinality of deleted attributes increases. According to IUA, it follows that time consuming may decrease. 


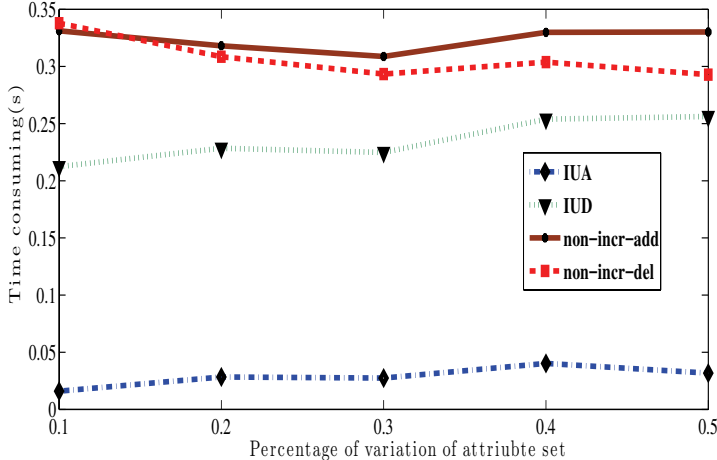

(a)heart-disease(cleveland).

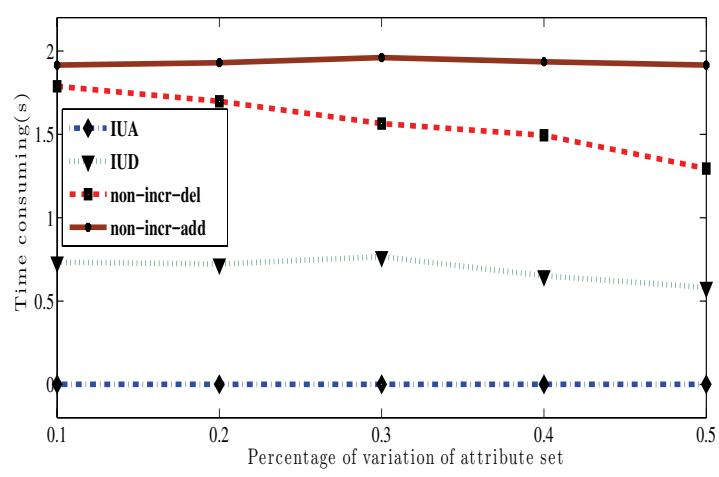

(c)arrhythmia

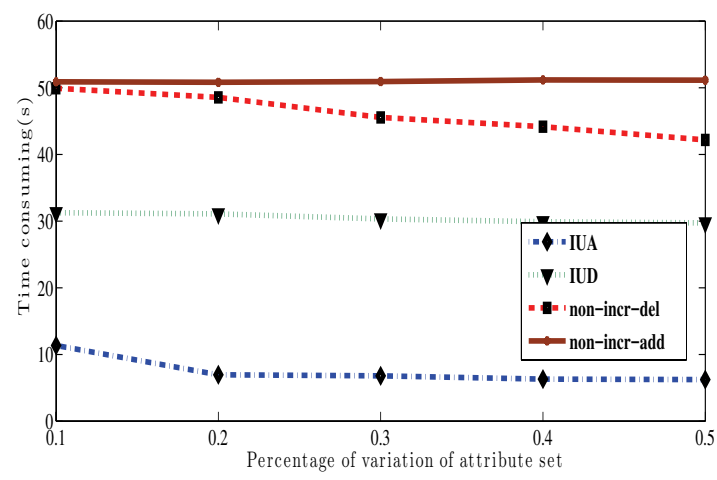

(e)statlog (Landsat Satellite)

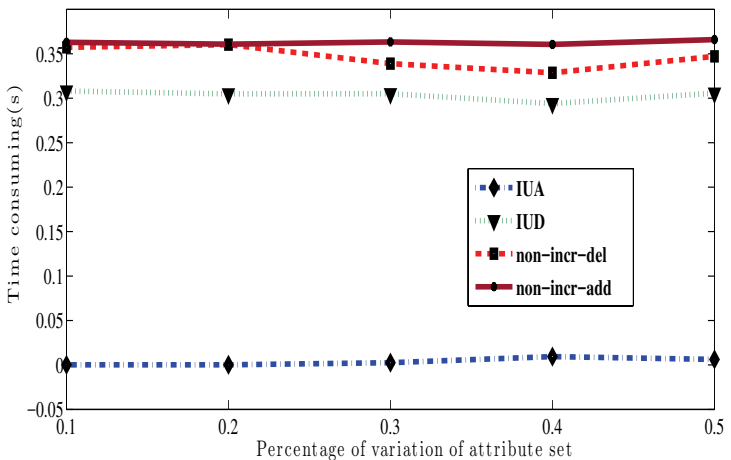

(b)lonosphere

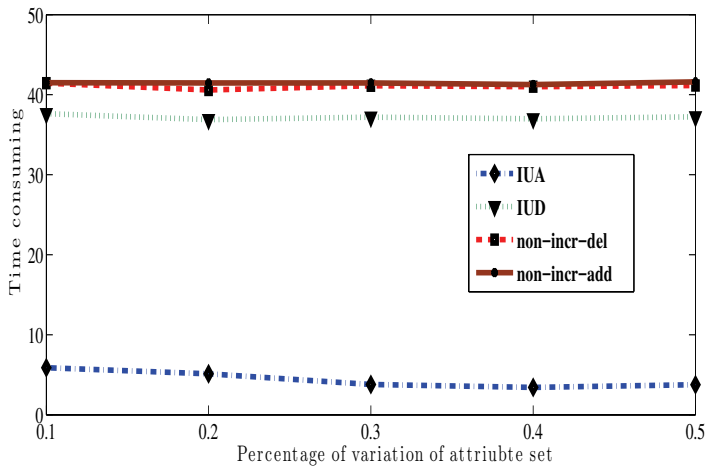

(d)winequality(white)

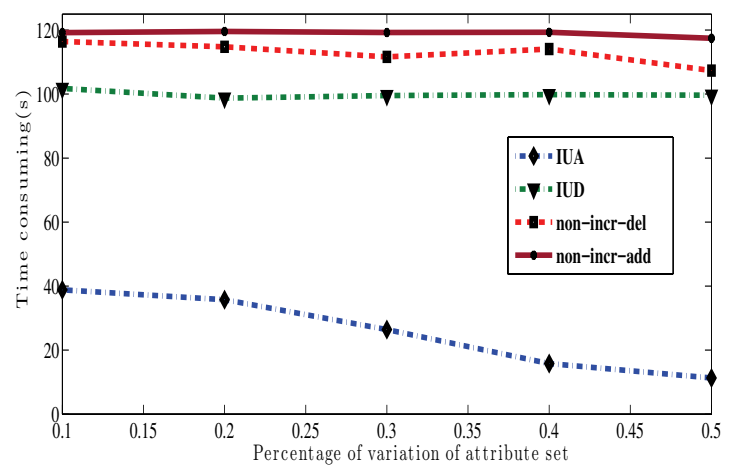

(f)mushroom

Fig. 1. A comparison of incremental and non-incremental of computing approximations under the variation of attribute set 


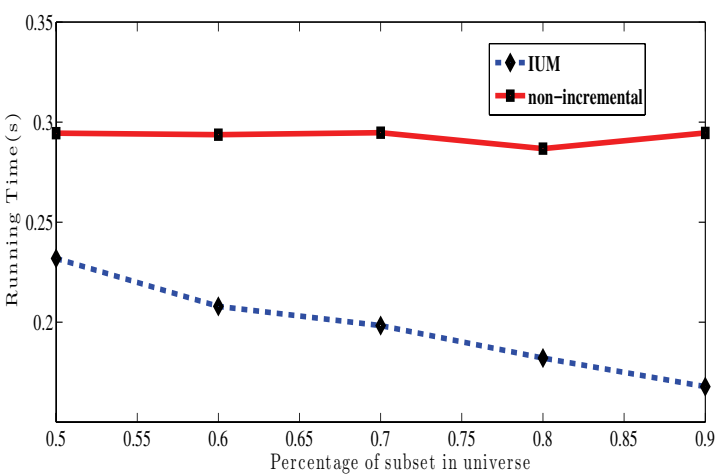

(a)heart-disease(cleveland).

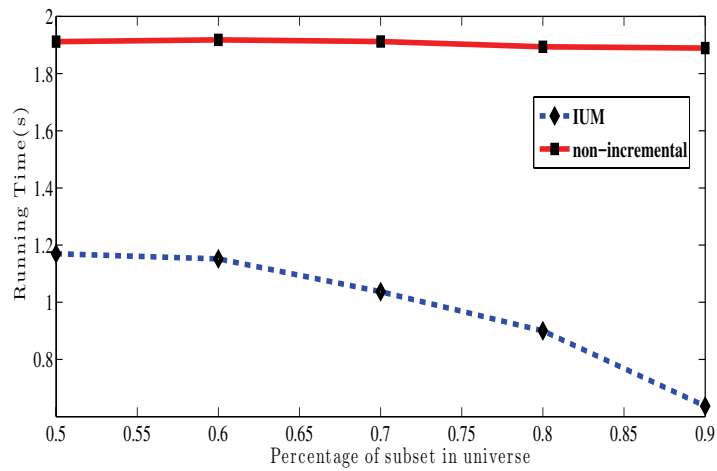

(c)arrhythmia

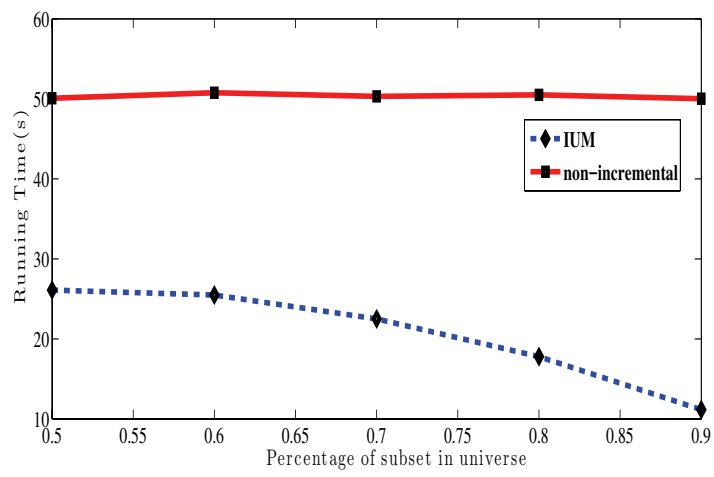

(e)statlog (Landsat Satellite)

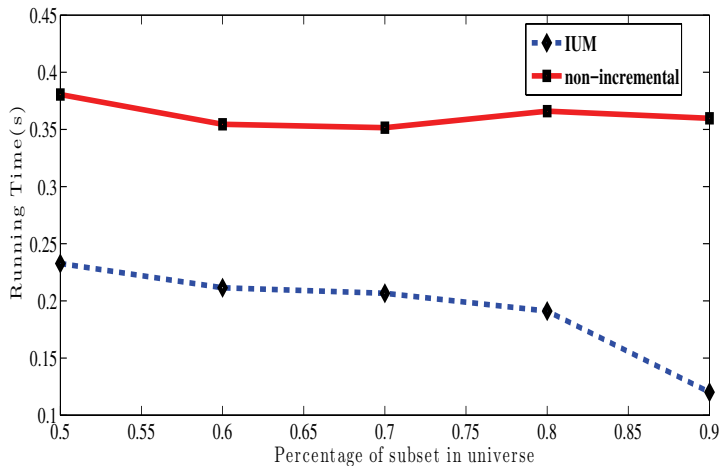

(b)lonosphere

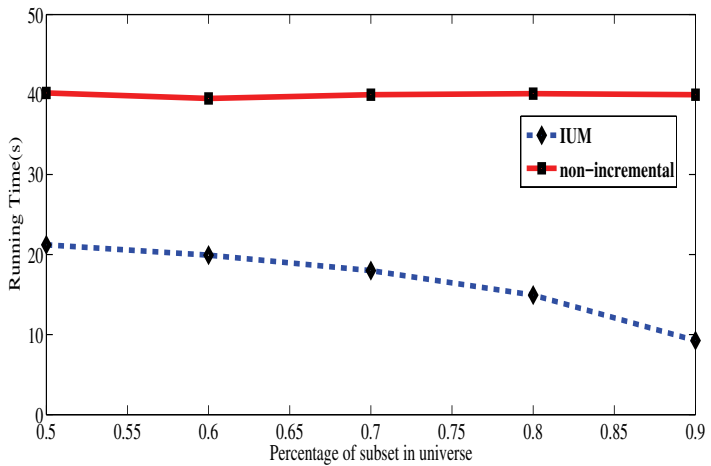

(d)winequality(white)

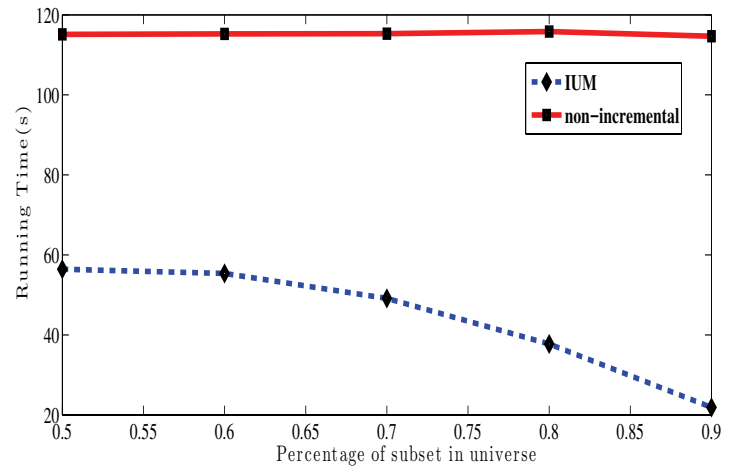

(f)mushroom

Fig. 2. A comparison of incremental and non-incremental of updating approximations when two subsets of objects are merged 


\subsection{Two subsets are merged}

For this section, we focus on update approximations when two subsets are merged. Analogously, time consuming of computation is recorded to compare the incremental approach IUM with nonincremental method.

The strategy of the experiment is the universe of each data sets are divided into two subsets. A group experiments are conducted, one subset is partitioned in percentage of universe from $50 \%$ to $90 \%$ and the other is remaining.

The experimental results of the six data sets are shown in Figure 2. In each of sub-figures, the $x$ coordinate pertains to percentage of the one subset in the universe, and $y$-coordinate pertains the time consuming of updating approximations when the other subset is combined into the one.

Clearly, from Figure 2(a)-(f), IUM is more effective than non-incremental approach when two subsets are combined into the universe. And the trend of time consuming of IUM goes down when percentage of the one subset in the universe increases.

\section{Conclusion and future work}

An information system evolves commonly in the dynamic data environment. It is important to accelerate the calculation of approximations in CDRSA in such dynamic environment. In this paper, we focus on the incremental approaches while the variation of attributes or the objects in IODS. The properties of confidential dominating/dominated sets under the variation of attributes or objects are discussed. Furthermore, incremental approaches of updating approximations are proposed when attributes or objects are dynamic changed. With the result of experiment, incremental approaches of updating approximations of CDRSA are feasible and effectively reduce the time consuming.

Our future work will focus on speeding up these approaches on the distributed computing environment for massive data sets.

\section{Ackownledgements}

This work is supported by the National Natural Science Foundation of China (Nos. 61272060 and 61572091), National key research and development program of China (No. 2016YFB1000905), Scientific and Technological Research Program of Chongqing Municipal Education Commission(No. KJ1600933), and Scientific Research Foundation of Sichuan Provincial Education Department (No. 16ZA0329)

\section{References}

1. Z. Pawlak, Rough sets. International Journal of Computer and Information Sciences, 11(5), (1982) 341356.

2. J. Li, Y. Ren, C. Mei, et al., A comparative study of multigranulation rough sets and concept lattices via rule acquisition, Knowledge-Based Systems, 85, (2015) 1-23.

3. C. H. Weng and C. K. Huang, Knowledge discovery of customer purchasing intentions by plausiblefrequent itemsets from uncertain data, Applied Intelligence, 43(3), (2015) 598-613.

4. T. R. Li, D. Ruan, Y. J. Shen, et al., A new weighting approach based on rough set theory and granular computing for road safety indicator analysis, Сотриtational Intelligence, 32(4), (2016) 517-534.

5. R. Bello and J. L. Verdegay, Knowledge Engineering for Rough Sets Based Decision-Making Models, International Journal of Intelligent Systems, 29(9), (2014) 823-835.

6. J. T. Yao and N. Azam, Web-Based Medical Decision Support Systems for Three-Way Medical Decision Making With Game-Theoretic Rough Sets, IEEE Transactions on Fuzzy Systems, 23(1), (2015) 3-15.

7. T. R. Li, C. Luo, H. M. Chen, et al. (eds.), The Principles and Methodologies of Big Data Mining-From the Perspectives of Granular Computing and Rough Sets, (Science Press, Beijing, 2016) (In Chinese).

8. J. B. Zhang, Y. Zhu, Y. Pan, et al., Efficient Parallel Boolean Matrix Based Algorithms for Computing Composite Rough Set Approximations, Information Sciences, 329, (2016) 287-302.

9. L. Bellodi, M. Battaglia, M. Gasperini, et al., Rough approximation by dominance relations, International Journal of Intelligent Systems, 17(2), (2002) 153-171.

10. S. Greco, Matarazzo B and R. Słowinski, Rough sets methodology for sorting problems in presence of multiple attributes and criteria, European Journal of $\mathrm{Op}$ erational Research, 138(2), (2002) 247-259. 
11. J. Błaszczynski, S. Greco and Słowinski, Multicriteria classification : A new scheme for application of dominance-based decision rules, European Journal of Operational Research, 181(3), (2007) 1030-1044.

12. T. Lee, S. Yeung and C. Tsang, Rough sets and ordinal reducts, Soft Computing, 10(1), (2006) 27-33.

13. H. M. Chen, T. R. Li, Y. Cai, et al., Parallel attribute reduction in dominance-based neighborhood rough set, Information Sciences, 373, (2016) 351-368.

14. J. J. H. Liou and G. H. Tzeng, A Dominance-based Rough Set Approach to customer behavior in the airline market, Information Sciences, 180(11), (2010) 2230-2238.

15. S. Greco, B. Matarazzo and R. Słowinski, A New Rough Set Approach to Evaluation of Bankruptcy Risk, Operational Tools in the Management of Financial Risks, (Springer US, 1998), pp.121-136.

16. J. Karami, A. Alimohammadi and T. Seifouri, Water quality analysis using a variable consistency dominance-based rough set approach, Computers Environment and Urban Systems, 43(1), (2014) 25-33.

17. S. Greco, B. Matarazzo and R. Słowinski, Handling Missing Values in Rough Set Analysis of Multiattribute and Multi-criteria Decision Problems. In Zhong, N., Skowron, A., Ohsuga, S. (eds.) RSFDGrC 1999. LNCS (LNAI), vol. 1711, Springer, Heidelberg pp.146-157.

18. S. Greco, B. Matarazzo and R. Słowinski, Dealing with Missing Data in Rough Set Analysis of MultiAttribute and Multi-Criteria Decision Problems, Decision Making: Recent Developments and Worldwide Applications, (Springer US, 2000).

19. J. Błaszczynski, R. Słowinski, (eds.) and M. Szelag, Induction of Ordinal Classification Rules from Incomplete Data, Rough Sets and Current Trends in Computing, (Springer Berlin Heidelberg, 2012), pp.56-65.

20. Y. Q. He and S. S. Hu, Rough analysis method of multi-attribute decision making with incomplete information, Journal of Systems Engineering, 19(2), (2004) 44-57. (in Chinese)

21. M. W. Shao and W. X. Zhang, Dominance relation and rules in an incomplete ordered information system, International Journal of Intelligent Systems, 20(1), (2005) 13-27.

22. M. L. Hu and S. F. Liu, An Analysis Method of Rough Multi-attribute Decision Making Based on Limited Extended Dominance Relation, Systems Engineering, 24(4), (2006) 106-110. (in Chinese)

23. M. L. Hu and S. F. Liu, Rough analysis method of multi-attribute decision making based on generalized extended dominance relation, Control and Decision, 22(12), (2007) 1347-1351. (in Chinese)

24. H. M. Chen, T. R. Li and D. Ruan, Maintenance of approximations in incomplete ordered decision systems while attribute values coarsening or refining,
Knowledge-Based Systems, 31(7), (2012) 140-161.

25. W. S. Du and B. Q. Hu, Dominance-based rough set approach to incomplete ordered information systems, Information Sciences, 346-347, (2016) 106-129.

26. X. B. Yang, J.Y.Yang, C. Wu et al., Dominancebased rough set approach and knowledge reductions in incomplete ordered information system, Information Sciences, 178 (2008) 1219-1234.

27. G. Z. Luo, X. J. Yang and D. Q. Zhou, Rough Analysis Model of Multi-attribute Decision Making Based on Limited Dominance Relation, Chinese Journal of Management Science, 18(4), (2009) 391-396. (in Chinese)

28. X. B. Yang and H. L. Dou, Valued DominanceBased Rough Set Approach to Incomplete Information System, Data and Knowledge Engineering, 68(11), (2011) 1331-1347.

29. G. L. Gou, G. Y. Wang, J. Li et al., Confidential dominance relation based rough approximation model, Control and Decision, 29(7), (2014) 1325-1329. (in Chinese)

30. C. C. Chan. A rough set approach to attribute generalization in data mining, Information Sciences, 107(14), (1998) 169-176.

31. T. R. Li , D. Ruan, W. Geert, et al. A rough sets based characteristic relation approach for dynamic attribute generalization in data mining, Knowledge-Based Systems, 2007, 20(5):485-494.

32. J. B. Zhang, T. R. Li, D. Ruan, et al., Rough sets based matrix approaches with dynamic attribute variation in set-valued information systems, International Journal of Approximate Reasoning, 53(4), (2012) 620-635.

33. S. Y. Li , T. R. Li and D. Liu, Incremental updating approximations in dominance-based rough sets approach under the variation of the attribute set, Knowledge-Based Systems, 40(1), (2013) 17-26.

34. Y. Y. Zhang, T. R. Li, C. Luo, et al., Incremental Updating of Rough Approximations in Interval-valued Information Systems under Attribute Generalization, Information Sciences. 373, (2016) 461-475.

35. D. Liu, T. R. Li and J. B. Zhang. Incremental updating approximations in probabilistic rough sets under the variation of attributes, Knowledge-Based Systems, 73, (2015) 81-96.

36. X. B. Yang, Y. Qi, H. L. Yu, et al., Updating multigranulation rough approximations with increasing of granular structures, Knowledge-Based Systems, 64, (2014) 59-69.

37. N. Shan and W. Ziarko, Data-based acquisition and incremental modification of classification rules, Computational Intelligence, 11(2), (1995) 357-370.

38. Z. Zheng and G. Y. Wang, RRIA: A rough set and rule tree-based incremental knowledge acquisition algorithm, Fundamenta Informaticae, 59(3), (2004) 299313. 
39. J. B. Zhang, T. R. Li, D. Ruan, et al., A parallel method for computing rough set approximations, Information Sciences, 194(5), (2012) 209-223.

40. J. B. Zhang, T. R. Li, D. Ruan, et al., Neighborhood rough sets for dynamic data mining, International Journal of Intelligent Systems, 27(4), (2012) 317-342.

41. C. Luo, T. R. Li, Z. Yi, et al., Matrix approach to decision-theoretic rough sets for evolving data, Knowledge-Based Systems, 99, (2016) 123-134.

42. C. Luo, T. R. Li, H. M. Chen, et al., Efficient updating of probabilistic approximations with incremental objects, Knowledge-Based Systems, 109, (2016) 71-83.

43. S. Y. Li, T. R. Li and J. Hu, Update of approximations in composite information systems, Knowledge-Based Systems, 83, (2015) 138-148.

44. S. Y. Li, T. R. Li and D. Liu, Dynamic Maintenance of Approximations in Dominance-Based Rough Set Approach under the Variation of the Object Set, International Journal of Intelligent Systems, 28(8), (2013) 729-751.

45. S. Y. Li, T. R. Li, Z Zhang, et al., Parallel computing of approximations in dominance-based rough sets approach, Knowledge-Based Systems, 87, (2015) 102-
111.

46. J. Błaszczynski and R.Słowinski, Incremental induction of decision rules from dominance-based rough approximations, Electronic Notes in Theoretical Computer Science, 82(4), (2003) 40-51.

47. S. Wang, T. R. Li, C. Luo, et al., Efficient updating rough approximations with multi-dimensional variation of ordered data, Information Sciences, 372, (2016) 690-708.

48. A. P. Zeng, T. R. Li, J. Hu, et al., Dynamical updating fuzzy rough approximations for hybrid data under the variation of attribute values, Information Sciences, 378, (2017) 363-388.

49. H. M. Chen, T. R. Li, D. Ruan, et al., A roughset-based incremental approach for updating approximations under dynamic maintenance environments, IEEE Transactions on Knowledge and Data Engineering, 25(2), (2013) 274-284.

50. M. Lichman,, UCI Machine Learning Repository [http://archive.ics.uci.edu/ml]. Irvine, CA: University of California, School of Information and Computer Science, (2013). 